\title{
An Animal Model of Genetic Vulnerability to Behavioral Disinhibition and Responsiveness to Reward-Related Cues: Implications for Addiction
}

\author{
Shelly B Flagel*,', Terry E Robinson², Jeremy J Clark, Sarah M Clinton', Stanley J Watson', Phillip Seeman ${ }^{4}$, \\ Paul EM Phillips ${ }^{3}$ and Huda Akil' \\ 'Molecular and Behavioral Neuroscience Institute, University of Michigan, Ann Arbor, MI, USA; ${ }^{2}$ Department of Psychology and Neuroscience \\ Program, University of Michigan, Ann Arbor, MI, USA; ${ }^{3}$ Department of Psychiatry and Behavioral Sciences and Pharmacology, University of \\ Washington, Seattle, WA, USA; ${ }^{4}$ Department of Pharmacology, University of Toronto, Toronto, ON, Canada
}

Rats selectively bred based on high or low reactivity to a novel environment were characterized for other behavioral and neurobiological traits thought to be relevant to addiction vulnerability. The two lines of animals, which differ in their propensity to self-administer drugs, also differ in the value they attribute to cues associated with reward, in impulsive behavior, and in their dopamine system. When a cue was paired with food or cocaine reward bred high-responder rats (bHRs) learned to approach the cue, whereas bred low-responder rats (bLRs) learned to approach the location of food delivery, suggesting that bHRs but not bLRs attributed incentive value to the cue. Moreover, although less impulsive on a measure of 'impulsive choice', bHRs were more impulsive on a measure of 'impulsive action' ie, they had difficulty withholding an action to receive a reward, indicative of 'behavioral disinhibition'. The dopamine agonist quinpirole caused greater psychomotor activation in bHRs relative to bLRs, suggesting dopamine supersensitivity. Indeed, relative to bLRs, bHRs also had a greater proportion of dopamine D2 ${ }^{\text {high }}$ receptors, the functionally active form of the receptor, in the striatum, in spite of lower D2 mRNA levels and comparable total D2 binding. In addition, fast-scan cyclic voltammetry revealed that bHRs had more spontaneous dopamine 'release events' in the core of the nucleus accumbens than bLRs. Thus, bHRs exhibit parallels to 'externalizing disorders' in humans, representing a genetic animal model of addiction vulnerability associated with a propensity to attribute incentive salience to reward-related cues, behavioral disinhibition, and increased dopaminergic 'tone.'

Neuropsychopharmacology (20I0) 35, 388-400; doi:I0.1038/npp.2009. I42; published online 30 September 2009

Keywords: novelty-seeking; sign tracking; goal tracking; impulsivity; dopamine; D2 ${ }^{\text {high }}$ receptor

\section{INTRODUCTION}

In humans substance abuse and addiction are often associated with a host of other behavioral syndromes collectively labeled 'externalizing disorders', which include impulsive and conduct disorders, in contrast to 'internalizing disorders', which include mood disorders (Kendler et al, 1997; Krueger et al, 2007). In addition to impulsivity, other traits that have been associated with addiction liability include novelty-seeking, sensation-seeking, and risk-taking behavior. These personality traits are often more broadly considered to reflect 'behavioral undercontrol' or 'behavioral disinhibition' (Dawe et al, 2004; Ball, 2005), and there is evidence linking such traits expressed early in childhood

*Correspondence: Dr SB Flagel, Molecular and Behavioral Neuroscience Institute, University of Michigan, 205 Zina Pitcher Place, Ann Arbor, MI 48I09, USA, Tel: (734) 936-2033, Fax: (734) 647-4I30,

E-mail: sflagel@umich.edu

Received 16 June 2009; revised II August 2009; accepted II August 2009 to the development of addiction in adulthood (eg, Masse and Tremblay, 1997; Ayduk et al, 2000). Although constitutive differences in emotional reactivity may influence how individuals respond to various environmental stimuli, there is still much to learn about the relationship between genetic endowment, environmental factors, and the neurobiological mechanisms that increase addiction vulnerability. Moreover, few animal models have addressed the antecedents of drug abuse vulnerability by linking genetic, neural, and behavioral elements. In this study, we used a selective-breeding strategy to demonstrate that genetic endowment not only alters reactivity to a novel environment, but also the tendency to attribute incentive value to reward-related cues, the ability to inhibit actions, and the dopamine system - in ways that may be associated with substance abuse resilience or vulnerability.

Rats were selectively bred on the basis of locomotor reactivity to a novel environment. Selective breeding produced a line of bred high-responder (bHR) rats that show high levels of locomotor activity in response to placement into a novel environment, and a line of bred 
low-responder (bLR) rats that are relatively inhibited in a novel environment (Stead et al, 2006). In outbred HR/LR rats, this trait has been associated with increased propensity to self-administer drugs (Piazza et al, 1989) and an increase in the amount of drug intake (Piazza et al, 2000). Consistent with studies in outbred HR/LR rats (Piazza et al, 1989; Kabbaj et al, 2000), bHRs acquire cocaine self-administration more rapidly than bLR rats (Davis et al, 2008), they exhibit a greater corticosterone response to a mild stressor, and they express lower levels of glucocorticoid receptor mRNA in the hippocampus (Clinton et al, 2008). Importantly, our studies demonstrate that the phenotype is genetic in nature and highly predictable from one generation to the next (Stead et al, 2006). By the tenth generation of breeding, we could predict with over $95 \%$ certainty the classification of our animals as high or low responders based on their selectively bred line (see Supplementary Figure 1). With each generation of breeding, the phenotypic differences in reactivity to novelty have become more pronounced, and the variability has continued to decrease (see Supplementary Figure 1). Moreover, selective breeding has amplified many of the differences seen in outbred animals, and uncovered additional facets of their behavioral phenotype that appear directly relevant to the enhanced differences in drug abuse susceptibility.

In this study, bHR and bLR rats were used to examine an array of behavioral traits and neurobiological characteristics associated with addiction vulnerability. Locomotor response to novelty was assessed in all rats to confirm the selectively bred phenotype. In addition, we studied the incentive motivational properties of cues associated with both food and drug (cocaine) reward using a Pavlovian conditional approach procedure. Furthermore, performance on three different tests of 'impulsivity' was assessed: delay-discounting and probabilistic-choice tasks to assess so-called 'impulsive choice', and a differential reinforcement of low rates of responding (DRL) task to assess 'impulsive action' or behavioral disinhibition. A number of addiction-related behaviors are believed to at least be partially regulated by the mesotelencephalic dopamine system (eg, Di Chiara, 1998; Cardinal and Everitt, 2004; Wise, 2004; Berridge, 2006), so we probed this system by quantifying the psychomotor response to quinpirole (a D2/D3 agonist; Levant et al, 1993) and by measuring 'basal' dopamine D2 receptor mRNA levels in bHR and bLR rats using in situ hybridization, total dopamine D2 receptor binding in the striatum, and the proportion of $\mathrm{D} 2$ high receptors, the functionally active form of the $\mathrm{D} 2$ receptor (see Seeman et al, 2005). Finally, we also used fast-scan cyclic voltammetry (FSCV) to quantify phasic dopamine release events (transients) in the nucleus accumbens core (Aragona et al, 2008). We hypothesized that, relative to bLR rats, bHR rats would attribute more incentive value to reward-related cues, appear more impulsive, and be more responsive to dopaminergic drugs as a reflection of altered dopaminergic regulation.

\section{MATERIALS AND METHODS}

Detailed methods can be found in the Supplementary Material and cited references.

\section{Subjects}

A total of 260 male Sprague-Dawley rats from our selective breeding colony (Stead et al, 2006) were used. Procedures were approved by the University Committee on the Use and Care of Animals. Unless otherwise indicated, rats were pairhoused and kept on a $12 \mathrm{~h}$ light $/ 12 \mathrm{~h}$ dark cycle (lights on 0600 hours) with controlled temperature and humidity. Food and water were available ad libitum.

Locomotor response to novelty. Each generation of rats was screened for locomotor activity around 60 days of age (see Stead et al, 2006; Clinton et al, 2007). Novelty-induced locomotor data were also obtained for bHRs and bLRs from the S17 generation using an automated behavioral analysis system (CleverSys, Reston, VA; Flagel and Robinson, 2007).

\section{Pavlovian conditional approach.}

Food-unconditional stimulus: Goal- and cue-directed Pavlovian approach responses were assessed in rats from generations S13-S16 using equipment and procedures described previously (Flagel et al, 2007, 2009). Each Pavlovian training session consisted of 25 trials, in which an illuminated lever (conditional stimulus, CS) was inserted into the chamber for $8 \mathrm{~s}$, followed immediately by the delivery of a 45-mg food pellet (unconditional stimulus, US) into the food cup (the 'goal'), on a random interval $90 \mathrm{~s}$ schedule. Note that no response was required for the rat to receive reward. Rats in the 'random' groups received pseudorandom CS and US presentations. The following events were recorded using Med Associates software: (1) number of lever contacts, (2) latency to the first lever contact, (3) number of food cup entries during CS presentation, (4) latency to the first food cup entry following CS presentation, and (5) number of food cup entries during the inter-trial interval (ITI).

Cocaine-unconditional stimulus: The propensity to approach a cocaine-paired cue was examined using rats from the S13 generation. After catheters were implanted (Weeks, 1972; Crombag et al, 2000), Pavlovian training was conducted in standard Med Associates chambers. A plastic translucent insert that formed a short corridor around the location of the lever was equipped with photocells to allow for the automated recording of lever approaches (see Uslaner et al, 2008). Each Pavlovian training session consisted of six trials (CS-US pairings) occurring on a randomly varying interval with a mean of $900 \mathrm{~s}$. Each trial consisted of the $8 \mathrm{~s}$ lever-CS presentation paired with the non-contingent intravenous injection of $0.5 \mathrm{mg} / \mathrm{kg}$ cocaine (US). Rats were tested for 12 sessions. The number of approaches and the latency to approach the lever-CS was recorded. The probability to approach the lever-CS was calculated as the number of trials with approach/the total number of trials.

\section{Impulsive behavior.}

Delay-discounting task: Impulsive choice was examined in rats from the S13 generation using a delay-discounting task. Procedures were adapted from Evenden and Ryan (1996) and Winstanley et al (2006). Test chambers had two retractable levers on either side of the central food cup and 
a white cue light was located above the food cup. Following pre-training (see Supplementary Material), rats were exposed to sessions consisting of five blocks of 12 trials. For each block, the first six trials were forced-choice trials, in which only one lever (either the 'immediate' or 'delay') was extended. The last six trials were free-choice trials, in which rats were able to choose between the immediate and delay levers. Responses on the immediate lever resulted in immediate delivery of one food pellet and responses on the delay lever resulted in delivery of four food pellets at a specified delay interval. For the first 18 test sessions, the delay associated with each trial block was $0,10,20,40$, and $60 \mathrm{~s}$. However, because rats rarely chose the delay lever over the immediate lever at the longer intervals, the delays were changed from session 19 onward to $0,3,6,12$, and $24 \mathrm{~s}$. The number of omitted or aborted trials, the number of initiated trials, the number of successful trials, and the number of responses on either the immediate or delay lever were recorded.

Probabilistic-choice task: bHR and bLR rats from the S17 generation were tested using a probabilistic-choice task similar to that described by Cardinal and Howes (2005). For this task, responses on one lever (small/certain) resulted in the delivery of one food pellet and responses on the other lever (large/uncertain) resulted in the delivery of four food pellets only if a given probability was met. Each test session consisted of five blocks of 16 trials. For each block, the first eight trials were forced-choice trials, in which only one lever was extended pseudorandomly. The last eight trials of each block were free-choice trials, in which both levers were extended and the rat was able to choose between the large/ uncertain $v s$ the small/certain lever. For the first seven test sessions the probability associated with the large/uncertain lever for each trial block was $1,0.5,0.25,0.125$, and 0.0625 . However, because rats rarely chose the large/uncertain lever over the small/certain lever at the lower probabilities, the probabilities were changed from session 8 onward to $1,0.75$, $0.50,0.375$, and 0.25 . The number of omitted or aborted trials, the number of initiated trials, the number of successful trials, and the number of responses on either the large/uncertain or small/certain lever were recorded.

$D R L$ : The same rats used for the delay-discounting task were also used for the DRL task (adapted from Uslaner and Robinson (2006)). Test chambers were arranged such that there was just one lever located to either the right or left (counterbalanced) of the food cup and the lever remained extended for the duration of the sessions. Each $45 \mathrm{~min}$ session began with illumination of the house light and lever presentation. Rats were initially tested on a DRL-5-s schedule, in which a lever press resulted in illumination of the lever and pellet delivery only if at least $5 \mathrm{~s}$ had elapsed since the previous press. Following 5 days of training at DRL-5-s, the schedule was changed to DRL-10-s for 5 days, then to DRL-20-s for 5 days, and the last 5 days at DRL-30-s. The total number of lever presses and the total number of reinforcers (ie, successful lever presses) were recorded.

Response to quinpirole. Rats from the S17 generation received three doses of quinpirole (counterbalanced; 0.1, 0.3 , and $1.0 \mathrm{mg} / \mathrm{kg}$, i.p.) or three injections of vehicle $(0.9 \%$
$\mathrm{NaCL}$, i.p.). Behavior was videotaped during habituation and the post-injection period, and Clever Sys (Reston, VA) Drug Scan software was used to analyze video-recorded behavior (Flagel and Robinson, 2007; Flagel et al, 2008).

\section{Dopamine regulation in $b H R$ and bLR rats.}

Dopamine D2 receptor $m R N A$ expression: Bred rats from the $\mathrm{S} 14$ generation were killed under basal conditions (without any previous manipulation) and dopamine D2 receptor mRNA expression was quantified in the nucleus accumbens (bregma levels 1.7-1.0) and caudate putamen (bregma levels 1.7-0.7) using in situ hybridization histochemistry (Kabbaj et al, 2000; Flagel et al, 2007).

Dopamine D2 receptor binding: Dopamine D2 receptor binding was measured by the competition of dopamine with $\left[{ }^{3} \mathrm{H}\right]$ domperidone (Seeman et al, 2003). Brains were collected from bHRs and bLRs from the S17 generation under basal conditions, and the dorsal striatum (caudateputamen) was dissected and rapidly frozen. This tissue was later homogenized and used for the competitive binding assay, as described by Seeman et al (2003). At low concentrations, dopamine inhibited the binding of $\left[{ }^{3} \mathrm{H}\right]$ domperidone between 1 and $100 \mathrm{nM}$ dopamine, and the second phase of inhibition occurred above $100 \mathrm{nM}$ dopamine. These two phases correspond to the high-affinity state of the dopamine D2 receptor, D2 ${ }^{\text {high }}$, and the low affinity state of the dopamine D2 receptor, respectively. The demarcation between the two phases (Seeman et al, 2005; Briand et al, 2008) clearly and readily permitted the measurement of the $\mathrm{D} 2{ }^{\text {high }}$ component as a percent of the total amount of specific $\left[{ }^{3} \mathrm{H}\right]$ domperidone binding, as defined by the presence of $10 \mu \mathrm{M}$ S-sulpiride.

Transient dopamine events: bHRs and bLRs from the S18 generation were transported to the University of Washington (Seattle, Washington) and FSCV was used to examine the frequency of spontaneous dopamine transients in the core of the nucleus accumbens (see Supplementary Figure 2) of awake rats, using chronically implanted carbonfiber microelectrodes (Sandberg et al, 2008). Procedures were adapted from Phillips et al (2003). Recordings were made in standard Med Associate chambers and food-evoked dopamine release was obtained by delivering an unexpected food pellet at the outset of each recording session. Spontaneous dopamine transients were determined by analyzing the final $2 \mathrm{~min}$ of each 1 -h recording session, during which a food pellet was initially delivered. Peak food-evoked dopamine release was determined by examining a $4 \mathrm{~s}$ window after pellet delivery.

\section{General Statistics}

Linear mixed-effects models (Verbeke and Molenberghs, 2000) were used to analyze the behavioral data. The covariance structure was explored and modeled appropriately for each dependent variable and when significant main effects or interactions were detected, Bonferroni post hoc comparisons were carried out. Independent $t$-tests were used to examine phenotypic differences in acquisition of behavior for the delay-discounting task and for the neurobiological data. 


\section{RESULTS}

\section{Locomotor Response to Novelty}

To confirm the phenotype of the selectively bred offspring, all rats from the breeding colony were screened for their locomotor response to novelty around day 60 of life, before any other testing. All rats used in the present experiments were from generations S13 to S18 (the specific generation used for each experiment is indicated in the Materials and methods and the Results section). Locomotor activity scores for the bHR and bLR rats used here are given in Table 1 for each generation. Even when using a subset of the selectively bred population, the novelty-induced locomotor scores were similar across generations and displayed little variance (see Table 1).

Selectively bred rats from the $\mathrm{S} 17$ generation (bHR $n=34$; bLR $n=33$ ) were tested a second time around 90 days of age for their locomotor response to a novel environment using a video-based system that allowed for a more detailed analysis of locomotor activity, including distance traveled (Figure 1a) and the speed of each bout of locomotion (Figure 1b). Behavior was quantified in 10-min blocks over a $2 \mathrm{~h}$ period after placement into the test chamber (Figure 1). Analyses using a linear mixed-effects model revealed a significant overall effect of phenotype for distance traveled (Figure 1a; $\mathrm{F}_{(1,65)}=15.21, P<0.0001$ ) as well as an effect of time $\left(\mathrm{F}_{(10,65)}=35.37, P<0.0001\right)$ and a phenotype $\times$ time interaction $\left(\mathrm{F}_{(10,65)}=4.93, P<0.0001\right)$. bHRs traveled a

Table I Locomotor Activity Scores Across Generations for the bHR/bLR Rats Included in Each Experiment

\begin{tabular}{lcc}
\hline Generation & bHR & bLR \\
\hline SI3 & $948 \pm 21(n=21)$ & $259 \pm 21(n=21)$ \\
SI4 & $1080 \pm 32(n=14)$ & $237 \pm 22(n=19)$ \\
SI5 & $1006 \pm 21(n=12)$ & $266 \pm 16(n=12)$ \\
SI6 & $1175 \pm 39(n=20)$ & $230 \pm 21(n=20)$ \\
SI7 & $981 \pm 17(n=56)$ & $14 \mid \pm 8(n=55)$ \\
SI8 & $764 \pm 34(n=5)$ & $101 \pm 23(n=5)$
\end{tabular}

This table represents the average locomotor activity score \pm SEM for the rats used from each generation for the current set of studies. greater distance than bLRs for the first 70 min after being placed in the novel chamber (Figure 1a). In addition, the average speed of each bout of locomotion was also greater in bHRs relative to bLRs (effect of phenotype, $\mathrm{F}_{(1,65)}=18.50$, $P<0.0001$; effect of time, $\mathrm{F}_{(10,65)}=23.35, P<0.0001$; phenotype $\times$ time interaction $\left.\mathrm{F}_{(10,65)}=3.10, P=0.003\right)$, and this difference persisted throughout the $2 \mathrm{~h}$ test session (Figure 1b). Thus, bHRs locomoted the same distance as bLRs during the last $50 \mathrm{~min}$ of the test session, but differences in the speed of travel persisted throughout the test session (Figure 1b; bHR vs bLR, $P<0.03$ from 0 to $110 \mathrm{~min})$.

\section{Pavlovian Conditional Approach}

Food US. The effect of Pavlovian training using food as the US (ie, pairing a lever-CS with food delivery) was examined in four independent experiments using bHR and bLR rats from generations S13-S16. The results were qualitatively and statistically similar across generations, and therefore, for the sake of simplicity, the data for each replication were pooled in Figure 2 (bHR $n=43$; bLR $n=43$ ). For detailed statistics on these measures see Supplementary Table 1 . Figure 2 shows that in bHR rats CS-US pairing led to the development of a conditional response (CR) directed toward the lever-CS, ie, a 'sign-tracking' CR (Figure 2a-c). Thus, with training, bHRs exhibited a greater probability of approaching the lever-CS (Figure 2a), vigorously engaged the lever (Figure $2 \mathrm{~b}$ ), and did so with increasing rapidity (Figure 2c). In contrast, with training bLRs did not develop a sign-tracking $\mathrm{CR}$, but during CS presentation their behavior was directed toward the food cup or goal; ie, they learned a 'goal-tracking' CR (Figure 2d-f). With training, bLRs came to approach the food cup during the CS period with increasing probability (Figure 2d), and increasing vigor (Figure $2 \mathrm{e}$ ), and they did so more and more rapidly (Figure 2f). For all of these measures, there was a significant effect of phenotype $(P<0.0001)$, a significant effect of session $(P \leqslant 0.02)$, and a phenotype $\times$ session interaction $(P \leqslant 0.001)$. The fact that these CRs were acquired as a function of training in both bHR and bLR rats is indicated by a significant effect of session for all measures of signtracking in bHRs $(P<0.0001$, Figure $2 \mathrm{a}-\mathrm{c})$ and of goal tracking in bLRs $(P \leqslant 0.0001$, Figure $2 \mathrm{~d}-\mathrm{f})$. Furthermore, the
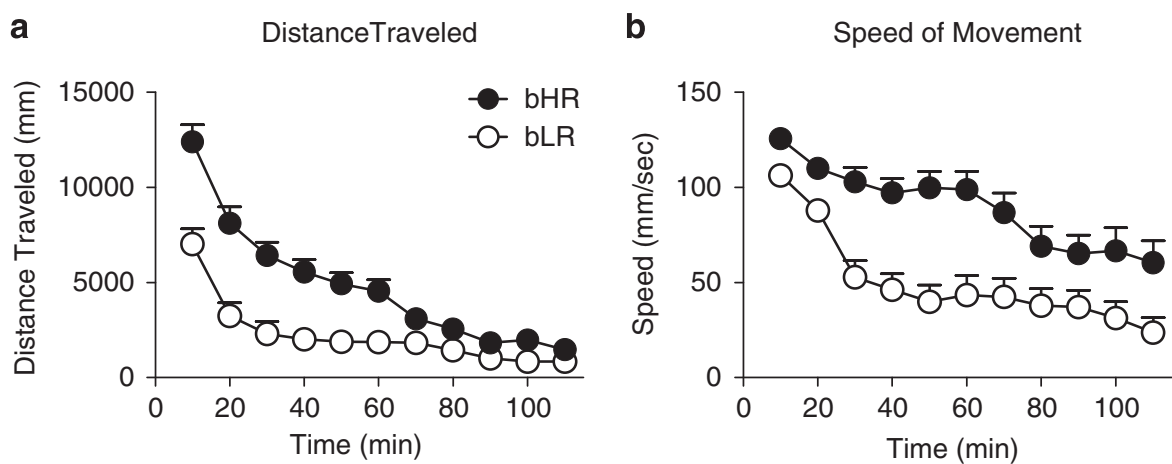

Figure I Time-course data for locomotor response to a novel environment in bHR $(n=34)$ and bLR $(n=33)$ rats. These data were obtained from automated analysis of video recordings and are represented as the mean + SEM for (a) distance traveled (in mm) and (b) speed of individual bouts of locomotion $(\mathrm{mm} / \mathrm{s})$. bHR and bLR rats traveled the same distance by the end of the habituation period, but continued to diverge in the speed of movement throughout the testing period. 


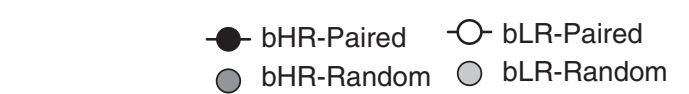

a
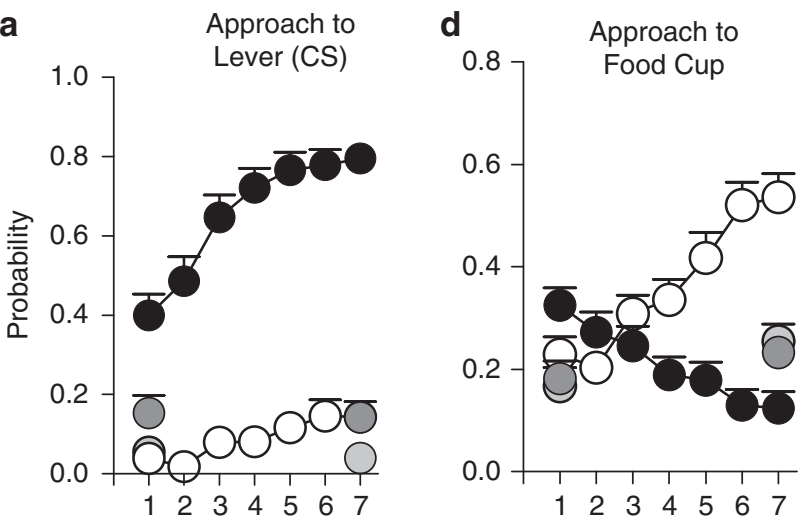

b

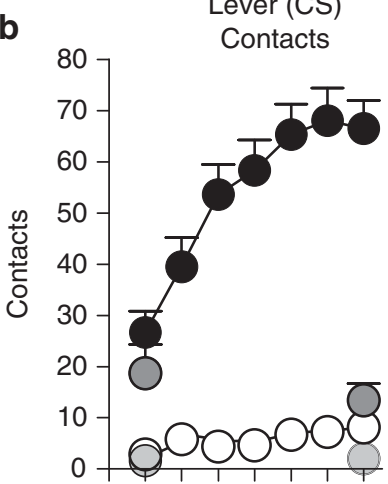

$\begin{array}{lllllll}1 & 2 & 3 & 4 & 5 & 6 & 7\end{array}$

C

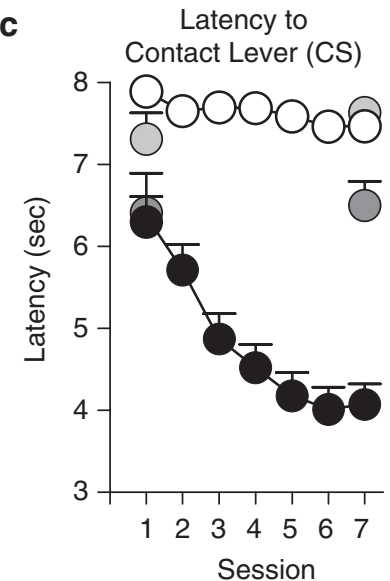

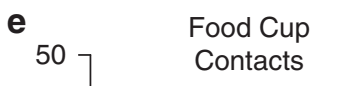

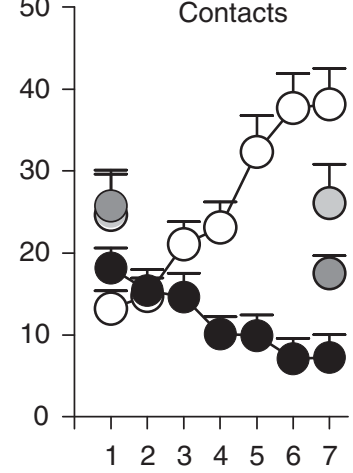

f

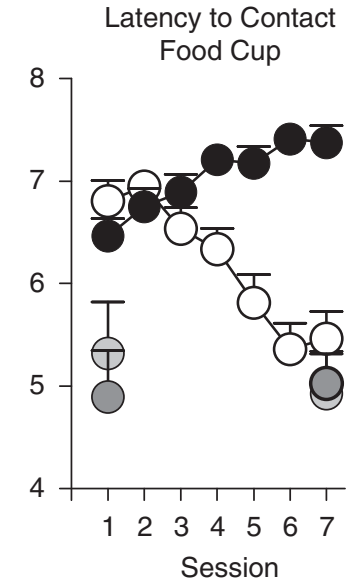

Figure 2 Comparison of CS-directed vs goal-directed conditional responses (CR) in bHR and bLR rats from generations $S|3-S| 6$. Rats in the 'paired' groups ( $n=43 /$ phenotype; bHR black circles; bLR open circles) received paired presentations of the lever-CS and food (US) and those in the random groups ( $n=1$ I/phenotype; bHR dark gray; bLR light gray) received pseudorandom presentations of the CS and US. Data are expressed as the mean + SEM and are illustrated over the course of seven training sessions (25 trials/session). The following variables are illustrated: (a) probability to approach the lever-CS; (b) number of contacts with the lever-CS; (c) latency to contact the lever-CS (in seconds, with 8 being maximum); (d) probability to approach the food cup; (e) number of contacts (or head entries) with the food cup; and (f) latency to contact the food cup during CS presentation. Both bHR and bLR rats (in the paired groups) learned a CR at about the same rate, as indicated by the changes in behavior over the course of training. However, for bHR rats, the CR was directed toward the CS-lever; whereas for bLRs it was directed toward the food cup.

development of both the sign-tracking and the goal-tracking $\mathrm{CR}$ was dependent upon the association between the CS and US, because rats that received random (unpaired) CS-US presentations did not develop either CR (session 1 vs session $7, P \geqslant 0.10$ for each phenotype on all measures; Figure 2a-f).

To better assess the rate of learning in bHR and bLR rats, we directly compared each of the three measures using analyses of variance, in which session was treated as a continuous variable. There were no significant differences between bHR and bLR rats in learning their respective CR, as indicated by nonsignificant group by session interactions for: (1) approach behavior; ie, comparing approach to the lever for bHRs $v s$ approach to the food cup for bLRs $\left(\mathrm{F}_{(1,589)}=0.26, P=0.61\right)$; (2) number of contacts with the lever-CS $v s$ the food cup $\left(\mathrm{F}_{(1,589)}=2.08, P=0.15\right)$; or (3) the latency to approach the lever-CS $v s$ the food cup $\left(\mathrm{F}_{(1,589)}=2.32, P=0.13\right)$. This analysis suggests that bHR and bLR rats not only learned their respective CRs, but they also did so at a comparable rate.

Finally, in one additional analysis we compared the difference in the probability of approaching the lever-CS $v s$ the food cup during the CS period across training sessions (ie, lever-food cup difference; Figure 3; see also Boakes, 1977; Flagel et al, 2009). The data presented in Figure 3 support the large bHR/bLR differences shown in Figure 2. For rats that received paired CS-US presentations there was a significant effect of phenotype $\left(\mathrm{F}_{(1,83)}=102.94\right.$, $P<0.0001)$, a significant effect of session $\left(\mathrm{F}_{(6,83)}=2.93\right.$, $P=0.01)$, and a phenotype $\times$ session interaction

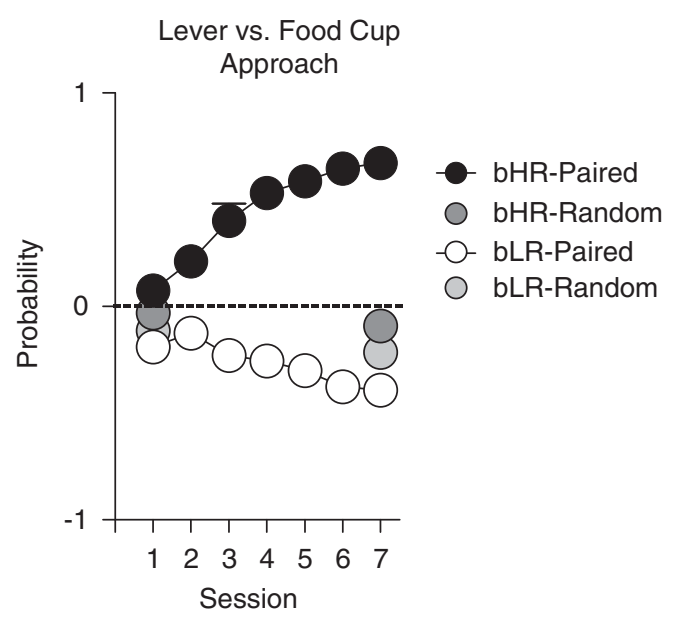

Figure 3 Difference in the probability of approach to the lever vs the food cup in selectively bred rats from generations SI3-SI6 (same as Figure 2; $n=43 /$ phenotype for paired groups, bHR-black circles; bLR-open circles; $n=11$ /phenotype for random groups, bHR - dark gray; bLR - light gray). Data are expressed as the mean + SEM probability of approach to the lever minus the probability of approach to the food cup. If the rat came into contact with the lever-CS on all 25 trials in a session and never made an entry into the food cup, it received a probability score of +1 . In contrast, a rat that on all trials made only food cup entries received a score of -1 . A score of zero (indicated by the dashed line) indicates that neither approach to the lever-CS nor approach to the food cup was dominant. bHR-paired rats developed a preference for CS-lever approach and bLR-paired rats exhibited a preference for goal-directed approach. The random groups did not develop a strong preference for either the lever-CS or the food cup. 
$\left(\mathrm{F}_{(6,83)}=12.43, P<0.0001\right)$. To determine whether bHR or bLR rats exhibited a preference for the lever or the food cup, respectively, one sample $t$-tests (with the hypothesized value of 0 ) were conducted. By the second training session, bHRpaired rats developed a clear preference for the lever-CS (score significantly $>0$ for sessions $2-7, P \leqslant 0.005$ ) and bLR-paired rats showed a preference for the food cup during all seven training sessions (score significantly $<0$, $P \leqslant 0.003)$. Figure 3 also shows that rats in the random groups tended to approach the food cup, but these animals did not show evidence of acquiring this response as a function of training (session 1 vs session $7, P \geqslant 0.12$ for each phenotype). It is likely that the preference for the food cup for bLR-paired rats and the random groups during the first training session was a result of 'pre-training', because all of the animals were required to retrieve pellets from the food cup during two pretraining sessions.

Taken together, these data indicate that bHR rats are primarily 'sign-trackers' (Hearst and Jenkins, 1974) because their CR consists of approach toward and engagement with the cue, or 'sign', that predicts reward delivery. On the other hand, bLR rats are primarily 'goal trackers' (Boakes, 1977) because upon CS presentation their CR consists of approach toward and engagement with the food cup (the 'goal'; see video files in Supplementary Material). In previous publications (Flagel et al, 2006, 2009; Robinson and Flagel, 2009), we have characterized outbred rats as sign trackers $v s$ goal trackers according to their CR (ie, the number of 'lever presses'). Sign trackers were defined as the one-third of animals that came to preferentially approach and vigorously engage the reward-predictive cue (ie, 'lever press' score in the top 33\%) and goal trackers as the one-third of animals that very rarely approached the reward-predictive CS, but instead learned to quickly go to the food cup (ie, lever press' score in the bottom 33\%). If the same criteria are applied for selectively bred rats from generations S13-S16, $100 \%$ of 'sign trackers' are bHR rats and $98 \%$ of 'goal trackers' are bLR rats. Thus, using the selectively bred lines, we can predict with almost $100 \%$ certainty whether an individual rat will be a sign tracker or a goal tracker. These findings are especially interesting because in outbred rats we found that the novelty-seeking trait and the sign-tracker/ goal-tracker traits are not correlated (Robinson and Flagel, 2009). It is possible, therefore, that these are independent traits, even in these selectively bred lines. To determine the extent to which they are genetically related will require additional studies, for example, by generating replicate or hybrid lines.

Cocaine US. Figure 4 shows the effect of pairing presentation of a lever-CS with an intravenous injection of cocaine (US) in bHR $(n=8)$ and bLR $(n=8)$ rats. With training, bHR rats developed a sign-tracking CR to the cocainepaired CS, whereas bLR rats did not. It is important to note that with a drug US there is no measurable goal-tracking response - that is, there is no specific location associated with delivery of the cocaine. Moreover, when cocaine is used as the US, animals rarely contact the lever-CS (as they do with a food US). Instead, the sign-tracking CR consists of orientation to the lever followed by approach and exploration (often sniffing) in the immediate vicinity of the lever, similar to that described when rewarding electrical brain stimulation is used as the US (see Peterson et al, 1972; Uslaner et al, 2006). Thus, CS-directed approach behavior is illustrated in Figure 4 as the probability that a rat approached the lever during the CS period in a given trial block (Figure $4 \mathrm{a}$ ), the number of approaches the rat made toward the lever (Figure $4 \mathrm{~b}$ ) and the latency with which the rat approached the lever (Figure 4c). For detailed statistics on these measures, see Supplementary Table 2. Relative to bLRs, with training bHRs increased their probability of approaching the lever-CS (Figure 4a), approached the leverCS more frequently (Figure $4 \mathrm{~b}$ ), and approached it with increasing rapidity (Figure $4 \mathrm{c}$ ). For all of these measures there was a significant effect of phenotype $(P \leqslant 0.01)$, a significant effect of trial block $(P=0.001)$, and a phenotype $\times$ trial block interaction $(P \leqslant 0.03)$. The fact that bHRs learned this CR is indicated by a significant effect of trial a

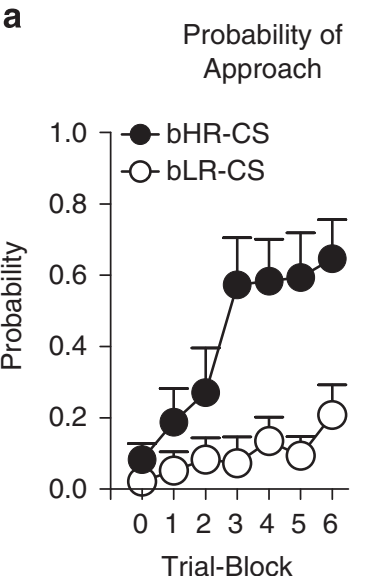

b

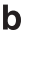

Approach to

Cocaine-Paired Cue

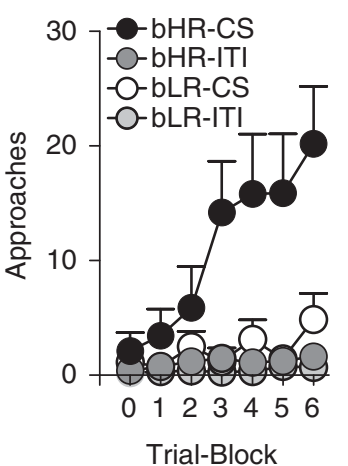

C
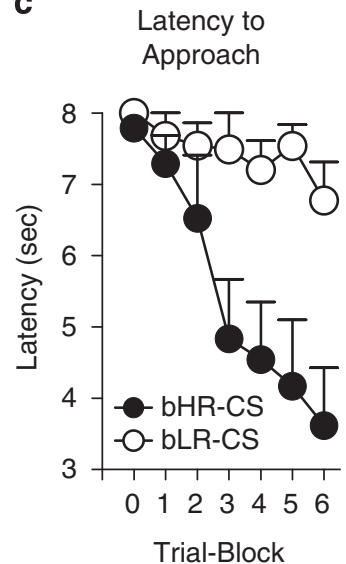

Figure 4 CS-directed approach to a cue associated with a non-contingent intravenous injection of cocaine in bHR ( $n=8$, black circles) and bLR ( $n=8$, open circles) rats. Data are illustrated as the mean + SEM averaged across I2-trial blocks. 'Block 0' represents baseline responding during the final habituation session. (a) The probability to approach the area of the cocaine-paired cue during lever-CS presentation. (b) The number of approaches to the lever-CS area during CS presentation (bHR-CS, bLR-CS) or during the ITIs (bHR-ITI, dark gray; bLR-ITI, light gray). (c) The latency to approach the cocaine-paired cue (in seconds, with 8 being maximum). bHR rats developed CS-directed approach (sign-tracking) to the cocaine-paired cue, and bLR rats did not. 
block when this group was analyzed independently for all measures $(P \leqslant 0.01)$. In contrast, for bLRs, the effect of trial block was not significant for any measure, indicating that they did not acquire this CR. Thus, bHRs learned to approach a CS paired with i.v. cocaine delivery, whereas bLRs did not.

Finally, Figure $4 \mathrm{~b}$ also shows that the phenotypic difference in approach to the cocaine-paired cue was not due to group differences in general exploratory behavior. Specifically, Figure $4 \mathrm{~b}$ illustrates the number of approaches to the lever-CS during CS presentation and during the ITIs. Behavior during the ITIs was converted into 8-s time bins to compare 'nonspecific' activity during the ITIs to that during the 8-s CS periods. There were no bHR/bLR differences during the ITI or during the baseline period (ie, trial block ' 0 '), indicating that, in this situation, the phenotypic differences emerged a result of Pavlovian training.

\section{Impulsive Behavior}

Delay-discounting task. Figure 5a shows the performance of bHR $(n=13)$ and bLR $(n=13)$ rats on the delaydiscounting task averaged across the last five test sessions (days 26-30). Lever press data (obtained during the freechoice trials) were converted into the percent of trials in which an animal chose the delayed reward by dividing the number of presses on the delay lever by the total number of lever presses for a given data block (maximum 6) and multiplying by 100 . bHRs chose the larger delayed reward significantly more often than bLRs (effect of phenotype, $\left.\mathrm{F}_{(1,25)}=9.95, P=0.004\right)$.

It is important to note that bHR rats acquired the behavior during the forced-choice training phase of this task at a faster rate than bLR rats (average number of days to meet criterion: bHRs $=7.1 \pm 1.7$, bLRs $=14.6 \pm 2.3$; $\mathrm{t}(24)=-2.7, P=0.01)$. We believe that these phenotypic differences are related to differences in performance- perhaps due to the differences in general activity levels- rather than in learning per se. Regardless, it is unlikely that these differences during training affected performance during the test sessions - especially since the data that were analyzed and reported were collected after weeks of exposure to the delay-discounting task. When performance deficits were examined during the test sessions (days 2630 ), bHR and bLR rats did not significantly differ on the total number of trials initiated (average bHR $=56.6 \pm 1.8$, $\mathrm{bLR}=51.9 \pm 1.8$; effect of phenotype, $\mathrm{F}_{(1,24)}=3.39, P=$ 0.08 ), the number of trials completed (average $\mathrm{bHR}=53.4 \pm 1.9, \quad \mathrm{bLR}=49.4 \pm 1.9$; effect of phenotype, $\left.\mathrm{F}_{(1,23)}=2.28, P=0.16\right)$, or the number of trials omitted (average bHR $=3.4 \pm 1.8, \mathrm{bLR}=8.2 \pm 1.8$; effect of phenotype, $\left.\mathrm{F}_{(1,24)}=3.39, P=0.08\right)$. Moreover, both phenotypes exhibited a similar response to the different delays (effect of delay $\mathrm{F}_{(4,63)}=41.53, P<0.0001$; phenotype $\times$ delay, $\mathrm{F}_{(4,63)}=$ $1.75, P=0.15)$.

Probabilistic-choice task. Figure $5 \mathrm{~b}$ shows the performance of bHR $(n=18)$ and bLR $(n=12)$ rats on the probabilisticchoice task averaged across the last five test sessions (days 20-24). Lever press data (obtained during the free-choice trials) were converted into the percent of trials in which an animal chose the larger probabilistic reward by dividing the number of presses on the probabilistic lever by the total number of lever presses for a given data block and multiplying by 100 . Interestingly, bHR and bLR rats performed almost identically on this task (ie, effect of phenotype, NS; phenotype $\times$ probability, NS). Moreover, bHRs and bLRs seemed equally capable of learning the probabilistic-choice task as there was an overall effect of probability $\left(\mathrm{F}_{(4,26)}=12.69, P<0.0001\right)$.

$D R L$. Figure 6 illustrates behavioral parameters indicative of 'impulsive action', as measured by the ability to withhold responding on a DRL task. On this task, bHRs $(n=13)$
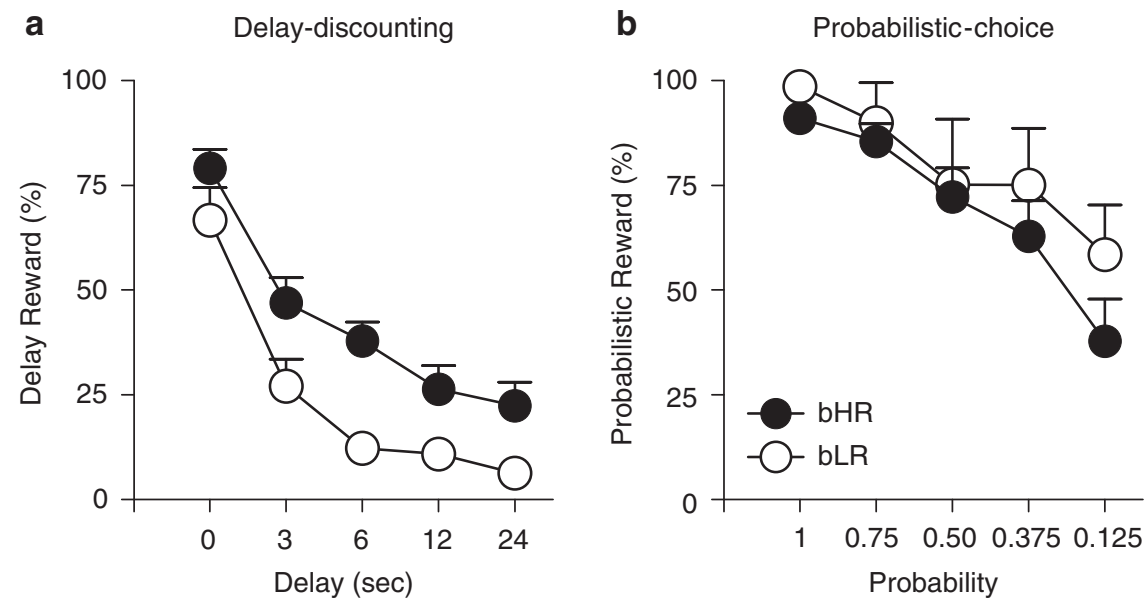

Figure 5 Performance on measures of 'impulsive choice'. (a) Performance on the delay-discounting task averaged across the last five test sessions (days 26-30) for bHR ( $n=13$, black circles) and bLR ( $n=13$, open circles) rats. Data represent the mean + SEM for the percent of trials on which the larger delayed reward was chosen and are illustrated for $0,3,6,12$, and $24 \mathrm{~s}$ intervals. bHR rats tended to choose the larger delayed reward more than bLR rats across delay intervals. (b) Performance on the probabilistic-choice task averaged across the last five test sessions (days $20-24)$ for bHR $(n=18)$ and bLR $(n=12)$ rats from SI7. Data represent the mean + SEM for the percent of trials on which the probabilistic lever was chosen at probabilities I, 0.75, 0.5, 0.375 , and 0.125 . bHR and bLR rats did not significantly differ on this task, but both groups showed a decrease in their choice for the larger reward with decreasing probabilities. 

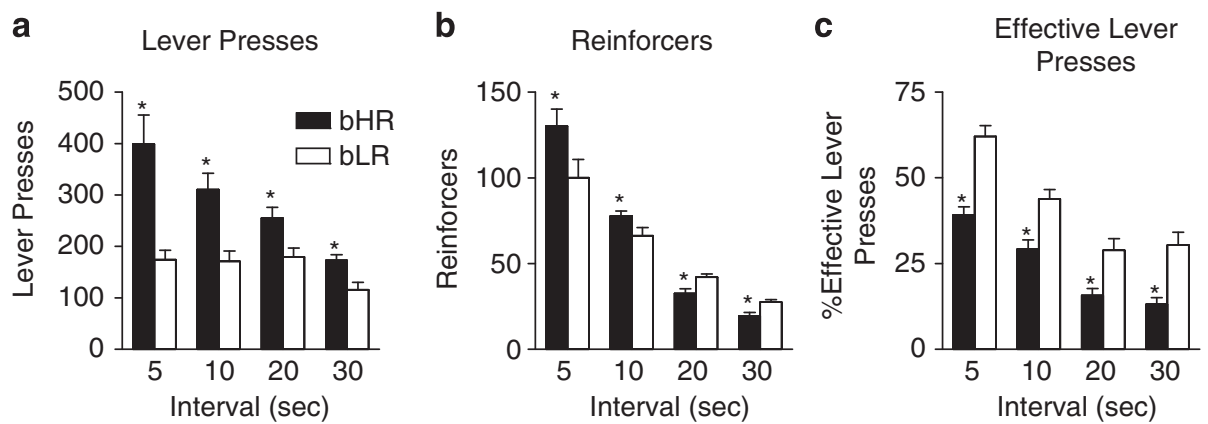

Figure 6 Performance on the DRL task, a measure of 'impulsive action.' Data are represented as the mean + SEM averaged across 5-day blocks for each DRL interval for bHR ( $n=13$, black circles) and bLR rats ( $n=13$, black circles). (a) The number of lever presses emitted. (b) The number of reinforcers received (ie, the number of successful lever presses). (c) The percentage of effective lever presses or the percent of lever presses that resulted in reward delivery. bHR rats were less able to withhold their response to receive a reward (ie, bHRs were more impulsive than bLRs on this task) and this effect was more pronounced at longer delay intervals. $* P \leqslant 0.05$ relative to $b L R$ at same delay interval.

performed more lever presses (Figure 6a: effect of phenotype, $\mathrm{F}_{(1,24)}=15, \quad P=0.001$; effect of delay, $\mathrm{F}_{(3,24)}=36.75, P<0.0001$; phenotype $\times$ delay, $\mathrm{F}_{(3,24)}=4.57$, $P=0.01)$ at all delay intervals, but received fewer reinforcers relative to bLRs $(n=13)$ at the two highest delay intervals (Figure 6b: effect of phenotype, $\mathrm{F}_{(1,24)}=1.82$, $P=0.19$; effect of delay, $\mathrm{F}_{(3,24)}=191.51, P<0.0001$; phenotype $\times$ delay, $\left.F_{(3,24)}=3.97, P=0.02\right)$. These results are better illustrated in Figure 6c, which shows 'effective lever presses', or the percent of lever presses that resulted in reward delivery. bLRs exhibited more effective lever presses than bHRs at all delay intervals (effect of phenotype, $\mathrm{F}_{(1,24)}=25.29, P<0.0001$; effect of delay, $\mathrm{F}_{(3,24)}=76.65$, $P<0.0001$; phenotype $\times$ delay, $\left.\mathrm{F}_{(3,24)}=2.4, P=0.09\right)$. The significant effect of delay for all of these measures suggests that both bHR and bLR rats are sensitive to the effect of delay, but it is more difficult for bHRs to withhold responding.

The same animals from the S13 generation were used for the delay-discounting task and the DRL task, so to insure there was no order effect we repeated just the DRL experiment using bHR and bLR rats from the S14 generation. When S14 rats were tested on the DRL task, we used nosepokes as the operant response rather than lever presses. Regardless, the data obtained using S14 rats (data not shown) were similar to those shown in Figure 6, supporting our conclusion that bHR rats have difficulty withholding actions relative to bLR rats.

\section{Response to Quinpirole}

The behavioral response to quinpirole, a direct agonist at D2/D3 dopamine receptors (Levant et al, 1993), was assessed in bHR ( $n=10-12 /$ group) and bLR ( $n=12$ /group) rats. Initial analysis of the time course of the behavioral effects indicated that the peak response fell between 25 and $55 \mathrm{~min}$ following injection, and therefore statistical analyses were conducted using data from this 30-min period. The effect of a saline injection was assessed in an independent group of animals and therefore these data were not included in the dose-effect analyses. However, independent $t$-tests were performed to determine whether the response at a specific dose significantly differed from that of the saline controls.
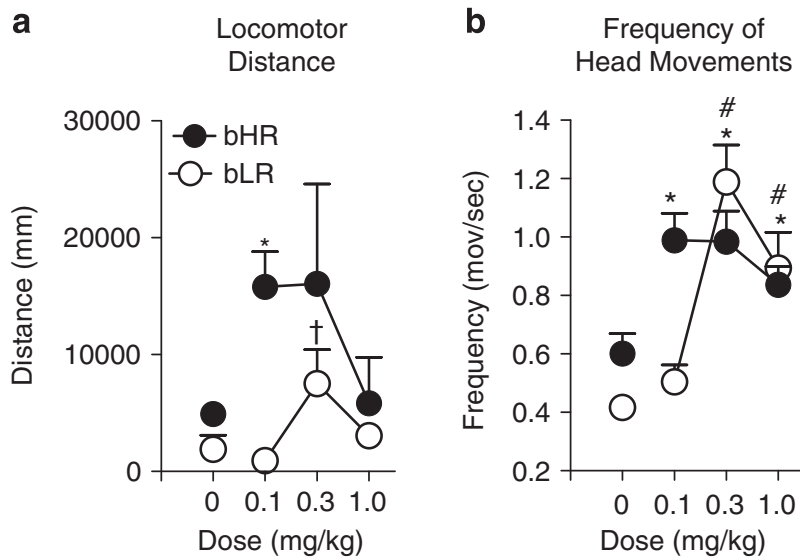

Figure 7 Psychomotor response to quinpirole. Data are illustrated as the mean + SEM for bHR $(n=12$, black circles $)$ and bLR $(n=12$, black circles) rats for each dose examined. Data illustrated at $0 \mathrm{mg} / \mathrm{kg}$ represent a separate control group of rats that received saline (bHR, $n=10 ; b L R$, $n=12$ ). (a) Locomotor distance traveled. (b) Frequency of head movements. * $P \leqslant 0.05$ bHR vs bHR saline; ${ }^{\#} P<0.05$ bLR vs bLR saline; ${ }^{\dagger} P<0.10$ bLR vs bLR saline.

Figure 7 illustrates the dose-response functions for locomotor distance and the frequency of head movements following quinpirole administration. There was a significant effect of phenotype for quinpirole-induced locomotor activity $\left(\mathrm{F}_{(1,23)}=9.99 ; P=0.01\right)$ and this effect was most pronounced at the lowest dose examined (Figure 7a). bHR rats exhibited a large increase in locomotor activity in response to $0.1 \mathrm{mg} / \mathrm{kg}$ of quinpirole relative to bLR rats and it was only at this lowest dose that bHR rats significantly differed from bHR saline control rats $(P=0.005)$. bLRs did not differ significantly from their saline control counterparts at any dose, but there was a trend level effect at $0.3 \mathrm{mg} / \mathrm{kg} \quad(P=0.09)$. There was also a trend for a phenotype $\times$ dose interaction $\left(\mathrm{F}_{(2,39)}=2.67, P=0.08\right)$, but no overall effect of dose. These results were likely affected by the large amount of variance for the bHRs at $0.3 \mathrm{mg} / \mathrm{kg}$, which was largely due to one animal that showed an unusually large locomotor response. Nevertheless, these data show that bHRs are more sensitive to a low dose of quinpirole than bLRs. 
The frequency of head movements was determined by dividing the total number of lateral head movements by the time spent in place, providing an index of the vigor of druginduced head movements when the animals are not engaged in other competing behaviors, such as locomotion (Flagel and Robinson, 2007; Flagel et al, 2008). There was no significant effect of phenotype for the frequency of head movements in response to quinpirole (Figure $7 \mathrm{~b}$ ), but there was a significant effect of dose $\left(\mathrm{F}_{(2,47)}=7.07, P=0.002\right)$ and a phenotype $\times$ dose interaction $\left(\mathrm{F}_{(2,47)}=7.40, P=0.002\right)$. Similar to locomotor activity, the bHR/bLR differences were most pronounced at the lowest dose examined $(P=0.001)$, which produced a greater increase in the frequency of head movements in bHRs than bLRs. For this measure bHR rats significantly differed from bHR saline rats at all three doses examined $(P \leqslant 0.01)$, and bLR rats differed from bLR saline rats only in response to the two highest doses examined $(P<0.002)$. Comparison of these responses revealed very different dose-effect functions for bHRs $v s$ bLRs. bLR rats exhibited an inverted U-shaped function, whereas bHR rats appeared to have reached their maximum response at the lowest dose tested. These data support the locomotor distance data, suggesting that bHRs are more sensitive to quinpirole than bLRs.

\section{Dopamine Regulation in bHR and bLR Rats}

Dopamine D2 receptor $m R N A$ expression. In situ hybridization was performed on brains obtained from bHR $(n=5)$ and bLR $(n=7)$ rats under 'basal' conditions; ie, the animals were not subjected to any manipulation. Dopamine D2 receptor mRNA levels were significantly lower in bHRs than bLRs in the nucleus accumbens (Figure $8 \mathrm{a}, P=0.04$ ), although this reached only a trend level of significance in the caudate-putamen (Figure 8b; $P=0.09$ ).

Dopamine D2 receptor binding. The total amount of dopamine $\mathrm{D} 2$ receptor binding and the proportion of $\mathrm{D} 2^{\text {high }}$ receptors were examined in the dorsal striatum (caudateputamen) of bHR $(n=5)$ and bLR $(n=5)$ rats. As a relative measure of total D2 receptor density, the total number of receptors occupied by $\left[{ }^{3} \mathrm{H}\right]$ domperidone was calculated (reflecting $81 \%$ of the total number of $\mathrm{D} 2$ receptors). The proportion of $\mathrm{D} 2{ }^{\text {high }}$ receptors was then determined by calculating the percentage of total binding representing the $\mathrm{D} 2{ }^{\text {high }}$ receptors. There were no group differences in the total number of D2 receptors (Figure $8 c, P=0.33$ ). However, relative to bLRs, bHRs had a significantly greater proportion $(\approx 30 \%$ more $)$ of $\mathrm{D} 2^{\text {high }}$ receptors in the dorsal striatum (Figure $8 \mathrm{~d}, t(8)=5.17, P=0.0009$ ).

Phasic dopamine activity. Fast-scan cyclic voltammetry was used to evaluate spontaneous and food-evoked phasic dopamine release in the core of the nucleus accumbens of bHR $(n=5)$ and bLR $(n=5)$ rats. The core of the nucleus accumbens was targeted because it has been implicated in a number of the behaviors reported here (for review see Everitt et al, 2008). The chemical identity of spontaneous and food-evoked events was assessed by statistically comparing the obtained signal to a template generated by electrical stimulation of the dopamine-containing cell bodies of the ventral tegmental area (Roitman et al, 2004). a

D2 mRNA NAcc

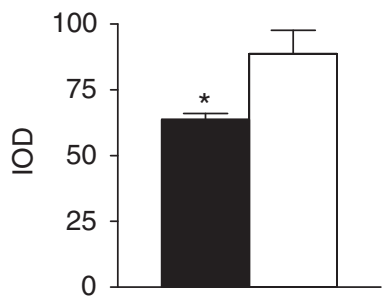

b

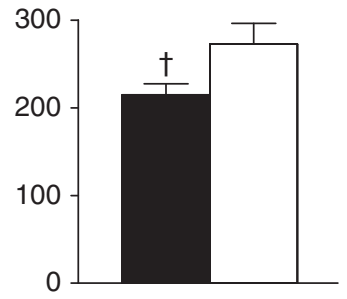

C

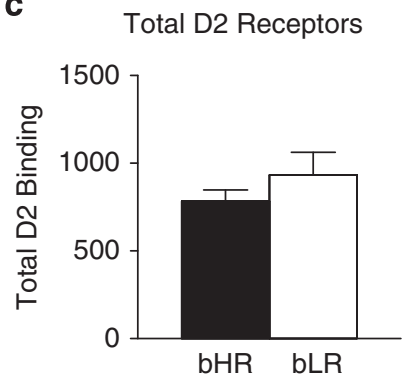

d

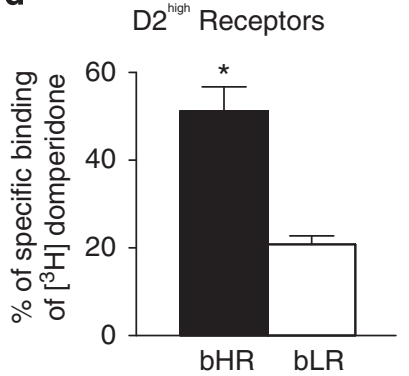

Figure 8 Dopamine D2 receptor profile in the striatum of selectively bred rat lines. ( $a$ and b) Data represent the mean + SEM of relative integrated optical density (IOD) for D2 mRNA in bHR $(n=5$, black bars) and $\operatorname{bLR}(n=7$, white bars) rats from SI4. (a) bHR rats exhibited lower levels of D2 mRNA in the nucleus accumbens (NAcc) and (b) a trend toward lower levels in the caudate putamen (CPu). (c) The mean + SEM of total dopamine receptors indicated by $\left[{ }^{3} \mathrm{H}\right]$ domperidone binding in the dorsal striatum of bHR ( $n=5$, black bars) and bLR $(n=5$, white bars) rats from S17. (d) The mean + SEM proportion of D2 ${ }^{\text {high }}$ receptors (in the same rats used in panel $c$ ). There was no significant difference in the total amount of $\mathrm{D} 2$ receptors, but bHR rats exhibited a greater proportion of $D 2^{\text {high }}$ receptors relative to bLR rats. ${ }^{*} P<0.05 ;{ }^{\dagger} P<0.10$ relative to bLR rats.
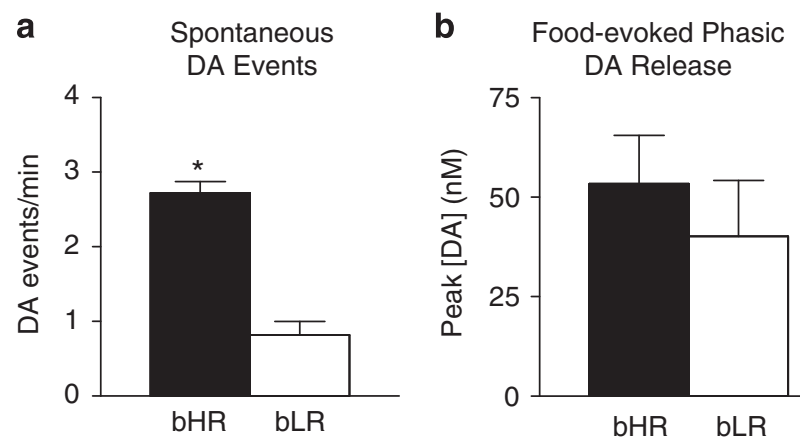

Figure 9 Phasic dopamine activity in the core of the nucleus accumbens. Data were obtained using in vivo fast-scan cyclic voltammetry in bHR $(n=5)$ and bLR $(n=5)$ rats. (a) Data represent the average number of spontaneous phasic dopamine events (+SEM). bHR rats showed a significantly greater number of spontaneous events in comparison with bLR rats. (b) Average peak dopamine response (+SEM) to food presentation in $b H R$ and $b L R$ rats. There were no significant differences between phenotypes in food-evoked dopamine response. ${ }^{*} P<0.00$ I relative to bLR rats.

Evoked changes in signal were attributed to dopamine if the associated cyclic voltammogram was closely correlated with that of stimulated release $\left(r^{2}>0.75\right)$. bHR rats showed significantly more spontaneous phasic dopamine events (transients) than bLR rats (Figure $9 \mathrm{a}, t(8)=8.01, P<0.001$ ). In contrast, there was no difference between phenotypes in 
the peak amplitude of dopamine release in response to the unconditional presentation of a food pellet (Figure 9b).

\section{DISCUSSION}

Two lines of rats were selectively bred for 13-18 generations based solely on whether they showed a high or low locomotor response after placement into a novel environment. We report here the extent to which these selectively bred lines differ on a number of other traits relevant to addiction vulnerability. Our studies highlight a complex array of phenotypic differences, including the tendency to attribute incentive salience to food- and cocaine-associated cues, impulsive behavior, and dopaminergic regulation.

\section{The Attribution of Incentive Salience to Reward-Related Cues}

It is thought that reward-related cues acquire the ability to maintain and instigate behavior in part because they acquire incentive motivational properties through Pavlovian learning, and cues associated with drugs may be attributed with pathological levels of incentive salience (Stewart et al, 1984; Robinson and Berridge, 1993; Everitt and Robbins, 2005; Tomie et al, 2008; Flagel et al, 2009). One property of stimuli attributed with incentive salience is that they become attractive, eliciting approach toward them. Using food as the US, we found that bHRs developed a signtracking CR, exhibiting approach and consummatory behavior directed toward the lever-CS. bLRs also acquired a CR, but it was not directed toward the CS, but to the location of reward delivery (ie, they learned a goal-tracking CR; see videos in Supplementary Material). Furthermore, when cocaine was used as the US only bHR animals developed a sign-tracking CR. Thus, a cue associated with either food or cocaine became attractive in bHR, but not bLR rats. This suggests that bHR animals, but not bLR animals, tend to attribute incentive salience to discrete stimuli predictive of reward (see Robinson and Flagel, 2009). This idea is supported by the finding that in outbred animals that show a sign-tracking CR the CS is also a more effective conditional reinforcer than it is for animals that show a goal-tracking CR (Robinson and Flagel, 2009), and we have found a similar difference between the bHR and bLR lines (unpublished data).

These findings are especially interesting because in outbred rats we found that the novelty-seeking trait and the sign-tracker/goal-tracker traits are not significantly correlated (Robinson and Flagel, 2009), suggesting that these two traits may be dissociable. It appears that the signtracker/goal-tracker trait was co-selected over the course of breeding for the novelty-seeking trait; however, to determine the extent to which the HR/LR and sign-tracker/ goal-tracker traits are genetically related will require additional studies, for example, the generation of replicate or hybrid lines.

\section{Impulsivity}

It has been suggested that robust sign-tracking behavior may reflect a lack of inhibitory control over behavior (Tomie, 1996; Tomie et al, 1998), which is one attribute of impulsivity. It is interesting, therefore, to compare differences in the selected lines in sign-tracking behavior with differences on various tests of 'impulsivity'. Of course, the concept of 'impulsivity' is multidimensional and it is thought that there are several distinct forms of so-called impulsive behavior (Evenden, 1999; Chamberlain and Sahakian, 2007). The preclinical drug abuse literature has focused primarily on two facets of impulsivity (Olmstead, 2006; Perry and Carroll, 2008)- 'impulsive choice' (ie, impulsive decision making) and 'impulsive action' (ie, impaired inhibition). 'Impulsive choice' was examined here using a delay-discounting procedure and a probabilistic-choice or risk-based decision-making task. Similar tasks have been used in clinical research and impairments on these tasks have been associated with externalizing disorders including substance abuse (eg, see Ersche et al, 2008; Bobova et al, 2009; de Wit, 2009).

Interestingly, bHRs were less impulsive than bLRs on the delay-discounting task, but there were no group differences on the probabilistic-choice task. Although we expected to see phenotypic differences in performance on these tasks, there is a growing body of literature suggesting that the underlying processes that mediate delay-discounting are dissociable from those that mediate the discounting of probabilistic rewards (for discussion see St Onge and Floresco, 2009). For example, a recent study suggests that the dopamine D2 receptor mediates risk-based decision making on the probabilistic-choice task (St Onge and Floresco, 2009), but activity at this receptor has not been shown to affect delay-discounting (van Gaalen et al, 2006). In light of these findings, it is somewhat surprising that we did not see a difference between bHR and bLR rats on the probabilistic-choice task. However, we speculate that behavior on these tasks would be differentially affected in bHR $v s$ bLR rats with the administration of dopaminergic drugs; further studies are underway to address this issue.

When 'impulsive action' was assessed using a DRL task, bHRs were more impulsive - they were less able to withhold responding to receive reward. These latter results are consistent with those recently reported in outbred HR/LR rats (Stoffel and Cunningham, 2008). However, another recent report using outbred rats and a different measure of impulsivity (ie, 5-CSRTT) suggests that impulsive behavior is not associated with the reactivity to novelty trait, and that it is 'impulsivity' rather than reactivity to a novel environment that is most relevant to compulsive drug use (Belin et al, 2008).

The fact that the different measures of impulsivity used here were dissociable is consistent with a number of other studies on impulsive action $v s$ impulsive choice (Seiden et al, 1979; Bradshaw and Szabadi, 1992; Cardinal et al, 2001; Uslaner et al, 2006). It is not clear why manipulations (selective breeding in this case) that increase impulsive action may sometimes also decrease impulsive choice, but a few hypotheses have been suggested (see Uslaner and Robinson, 2006). One possibility is that the internal pacemaker responsible for the timing of events is differentially regulated for bHRs vs bLRs, therefore altering performance on the delay-discounting and DRL tasks without affecting behavior on the probabilistic-choice task. However, close examination of the data suggests that both phenotypes are influenced by delay in a comparable way, 
making such an explanation unlikely. Alternatively, group differences in the attribution of incentive salience to stimuli may also affect performance on the 'impulsivity tasks.' bHRs may be less able to withhold responding on the DRL task because the manipulandum is imbued with greater incentive value, thus more readily instigating an action (Tomie, 1996). Likewise, relative to bLRs, bHRs may prefer larger but delayed rewards because the incentive value of the large reward is disproportionately enhanced relative to the small reward. Indeed, a lesion of the subthalamic nucleus (STN) produces a pattern of behavior very similar to that shown here for bHRs. Relative to control animals, rats with an STN lesion more readily acquire cocaine selfadministration behavior, show more robust sign-tracking behavior to both food- and cocaine-associated cues, have difficulty withholding actions on a DRL task, but are more likely to wait for a larger but delayed reward on a delaydiscounting task (Uslaner et al, 2005, 2008; Uslaner and Robinson, 2006) - similar to bHRs. Uslaner et al (2008) suggested that this pattern of behavior may all be a consequence of an increased tendency to attribute incentive salience to reward-related cues after STN lesions. If this is the case, it would be interesting to compare STN function in bHR and bLR rats.

\section{Dopamine}

This study revealed a number of differences in the dopamine system of bHRs $v s$ bLRs. Relative to bLRs, bHRs are more sensitive to the psychomotor-activating effects of the D2/D3 agonist, quinpirole. Moreover, bHRs have a greater proportion $\mathrm{D} 2{ }^{\text {high }}$ receptors (Seeman et al, 2005) in the dorsal striatum even though they have lower levels of D2 mRNA and do not differ on measures of total D2 receptor binding. At first glance these findings may seem incongruent, but they may actually help explain a longstanding paradox. Outbred HR rats are known to exhibit increased responsiveness to dopaminergic drugs including psychostimulants, but have lower levels of D2 mRNA and dopamine receptor binding relative to LR rats (Hooks et al, 1994). Our current findings suggest that an increased proportion of $\mathrm{D} 2^{\text {high }}$ receptors in bHRs may account for the behavioral hypersensitivity following treatment with quinpirole. In agreement, many treatments that elicit a functional supersensitivity to dopamine (eg, amphetamine sensitization, cocaine self-administration) produce a large increase in the proportion of $\mathrm{D} 2^{\text {high }}$ receptors in the striatum, even if they produce no change or a decrease in the total number of D2 receptors (Seeman et al, 2005, 2007; Briand et al, 2008). Reduced striatal D2 receptors have been reported in cocaine addicts (Volkow et al, 1993; Martinez et al, 2004), in non-human primates allowed to selfadminister cocaine (Nader et al, 2006), and in rats with an impulsivity trait known to predict high rates of cocaine self-administration (Dalley et al, 2007). In all of these imaging studies, however, the ligands used could not discriminate between the low- and high-affinity D2 receptor states. Thus, much of this seemingly disparate literature could potentially be explained by an increase in the proportion of $\mathrm{D} 2^{\text {high }}$ receptors.

We also found that bHRs exhibit a greater number of spontaneous dopamine release events compared with bLRs, using FSCV to detect phasic dopamine activity in the core of the nucleus accumbens. Interestingly, there were no bHR/ bLR differences in food-evoked dopamine release, suggesting that both phenotypes are equally responsive to the receipt of reward using this measure. These findings are consistent with a number of electrophysiological and dialysis studies using outbred HR/LR rats (Piazza et al, 1991; Hooks et al, 1992; Marinelli and White, 2000). Additional studies are underway to further investigate reward- and cue-evoked dopamine transients in the bHR/ bLR lines.

In summary, bHRs exhibit a number of characteristics related to addiction vulnerability and 'externalizing disorders' in humans. We had previously reported their increased propensity to risk-taking behavior (Stead et al, 2006; Clinton et al, 2008) and to drug self-administration (Davis et al, 2008). In this study we show that relative to bLR rats, bHR rats also exhibit (1) a greater tendency to attribute incentive salience to cues predictive of both food and cocaine; (2) poor inhibitory control over behavior (ie, increased impulsive action); and (3) a hypersensitive dopamine system. Importantly, these studies also establish that this cluster of traits is highly heritable. Beyond involvement of the dopaminergic system, the specific genes and neural circuits responsible for these different traits related to substance abuse liability remain to be elucidated. However, the development and characterization of a genetic animal model for behavioral undercontrol represents a key step in defining the biological causes of increased propensity to externalizing disorders, including addiction, and in identifying biomarkers and molecular targets for their treatment.

\section{ACKNOWLEDGEMENTS}

This study was supported by grants from the National Institute of Drug Abuse to TER (R37 DA04294) and HA (R01 DA012286) and a program project grant which includes both HA and TER (5P01DA021633-02). The selective breeding colony was supported by the Office of Naval Research to HA (N00014-02-1-0879). Funding was also provided by the Institute for Research on Pathological Gambling and Related Disorders (Cambridge Health Alliance), awarded to SBF. We thank Lisa Briand, Sharon Burke, Jennifer Fitzpatrick, Julia Garcia-Fuster, Sue Miller, Jessica Meves, Tracy Simmons, and Ashley Strongwater for technical assistance with these experiments.

\section{DISCLOSURE}

The authors declare no conflict of interest.

\section{REFERENCES}

Aragona BJ, Cleaveland NA, Stuber GD, Day JJ, Carelli RM, Wightman RM (2008). Preferential enhancement of dopamine transmission within the nucleus accumbens shell by cocaine is attributable to a direct increase in phasic dopamine release events. J Neurosci 28: 8821-8831.

Ayduk O, Mendoza-Denton R, Mischel W, Downey G, Peake PK, Rodriguez M (2000). Regulating the interpersonal self: strategic 
self-regulation for coping with rejection sensitivity. J Pers Soc Psychol 79: 776-792.

Ball S (2005). Personality traits, problems and disorders: clinical applications to substance use disorders. J Res Personality 39: 84-102.

Belin D, Mar AC, Dalley JW, Robbins TW, Everitt BJ (2008). High impulsivity predicts the switch to compulsive cocaine-taking. Science 320: 1352-1355.

Berridge KC (2006). The debate over dopamine's role in reward: the case for incentive salience. Psychopharmacology (Berl) 191: 391-431.

Boakes R (1977). Performance on learning to associate a stimulus with positive reinforcement. In: Davis $\mathrm{H}$, Hurwitz $\mathrm{H}$ (eds). Operant-Pavlovian Interactions. Hillsdale, NJ: Erlbaum. pp 67-97.

Bobova L, Finn PR, Rickert ME, Lucas J (2009). Disinhibitory psychopathology and delay discounting in alcohol dependence: personality and cognitive correlates. Exp Clin Psychopharmacol 17: 51-61.

Bradshaw CM, Szabadi E (1992). Choice between delayed reinforcers in a discrete-trials schedule: the effect of deprivation level. Q J Exp Psychol B 44: 1-6.

Briand LA, Flagel SB, Seeman P, Robinson TE (2008). Cocaine selfadministration produces a persistent increase in dopamine D2 High receptors. Eur Neuropsychopharmacol 18: 551-556.

Cardinal RN, Everitt BJ (2004). Neural and psychological mechanisms underlying appetitive learning: links to drug addiction. Curr Opin Neurobiol 14: 156-162.

Cardinal RN, Howes NJ (2005). Effects of lesions of the nucleus accumbens core on choice between small certain rewards and large uncertain rewards in rats. BMC Neurosci 6: 37.

Cardinal RN, Pennicott DR, Sugathapala CL, Robbins TW, Everitt BJ (2001). Impulsive choice induced in rats by lesions of the nucleus accumbens core. Science 292: 2499-2501.

Chamberlain SR, Sahakian BJ (2007). The neuropsychiatry of impulsivity. Curr Opin Psychiatry 20: 255-261.

Clinton S, Miller S, Watson SJ, Akil H (2008). Prenatal stress does not alter innate novelty-seeking behavioral traits, but differentially affects individual differences in neuroendocrine stress responsivity. Psychoneuroendocrinology 33: 162-177.

Clinton SM, Vazquez DM, Kabbaj M, Kabbaj MH, Watson SJ, Akil $\mathrm{H}$ (2007). Individual differences in novelty-seeking and emotional reactivity correlate with variation in maternal behavior. Horm Behav 51: 655-664.

Crombag HS, Badiani A, Maren S, Robinson TE (2000). The role of contextual versus discrete drug-associated cues in promoting the induction of psychomotor sensitization to intravenous amphetamine. Behav Brain Res 116: 1-22.

Dalley JW, Fryer TD, Brichard L, Robinson ES, Theobald DE, Laane $\mathrm{K}$ et al (2007). Nucleus accumbens D2/3 receptors predict trait impulsivity and cocaine reinforcement. Science 315: $1267-1270$

Davis BA, Clinton SM, Akil H, Becker JB (2008). The effects of novelty-seeking phenotypes and sex differences on acquisition of cocaine self-administration in selectively bred high-responder and low-responder rats. Pharmacol Biochem Behav 90: 331-338.

Dawe S, Gullo MJ, Loxton NJ (2004). Reward drive and rash impulsiveness as dimensions of impulsivity: implications for substance misuse. Addict Behav 29: 1389-1405.

de Wit H (2009). Impulsivity as a determinant and consequence of drug use: a review of underlying processes. Addict Biol 14: 22-31.

Di Chiara G (1998). A motivational learning hypothesis of the role of mesolimbic dopamine in compulsive drug use. J Psychopharmacol 12: 54-67.

Ersche KD, Roiser JP, Robbins TW, Sahakian BJ (2008). Chronic cocaine but not chronic amphetamine use is associated with perseverative responding in humans. Psychopharmacology (Berl) 197: $421-431$.
Evenden JL (1999). Varieties of impulsivity. Psychopharmacology (Berl) 146: 348-361.

Evenden JL, Ryan CN (1996). The pharmacology of impulsive behaviour in rats: the effects of drugs on response choice with varying delays of reinforcement. Psychopharmacology (Berl) 128: $161-170$

Everitt BJ, Belin D, Economidou D, Pelloux Y, Dalley JW, Robbins TW (2008). Neural mechanisms underlying the vulnerability to develop compulsive drug-seeking habits and addiction. Philos Trans $R$ Soc Lond B Biol Sci 363: 3125-3135.

Everitt BJ, Robbins TW (2005). Neural systems of reinforcement for drug addiction: from actions to habits to compulsion. Nat Neurosci 8: 1481-1489.

Flagel SB, Akil H, Robinson TE (2009). Individual differences in the attribution of incentive salience to reward-related cues: Implications for addiction. Neuropharmacology 56(Suppl 1): 139-148.

Flagel SB, Robinson TE (2007). Quantifying the psychomotor activating effects of cocaine in the rat. Behav Pharmacol 18: 297-302.

Flagel SB, Watson SJ, Robinson TE, Akil H (2006). An animal model of individual differences in 'conditionability': relevance to psychopathology. Neuropsychopharmacology 31: S262-S263.

Flagel SB, Watson SJ, Robinson TE, Akil H (2007). Individual differences in the propensity to approach signals vs goals promote different adaptations in the dopamine system of rats. Psychopharmacology (Berl) 191: 599-607.

Flagel SB, Watson SJ, Akil H, Robinson TE (2008). Individual differences in the attribution of incentive salience to a rewardrelated cue: influence on cocaine sensitization. Behav Brain Res 186: $48-56$.

Hearst E, Jenkins H (1974). Sign-tracking: the stimulus-reinforcer relation and directed action. In: Monograph of the Psychonomic Society. Austin, TX.

Hooks MS, Colvin AC, Juncos JL, Justice Jr JB (1992). Individual differences in basal and cocaine-stimulated extracellular dopamine in the nucleus accumbens using quantitative microdialysis. Brain Res 587: 306-312.

Hooks MS, Juncos JL, Justice Jr JB, Meiergerd SM, Povlock SL, Schenk JO et al (1994). Individual locomotor response to novelty predicts selective alterations in D1 and D2 receptors and mRNAs. J Neurosci 14: 6144-6152.

Kabbaj M, Devine DP, Savage VR, Akil H (2000). Neurobiological correlates of individual differences in novelty-seeking behavior in the rat: differential expression of stress-related molecules. J Neurosci 20: 6983-6988.

Kendler KS, Davis CG, Kessler RC (1997). The familial aggregation of common psychiatric and substance use disorders in the National Comorbidity Survey: a family history study. $\mathrm{Br} J$ Psychiatry 170: 541-548.

Krueger RF, Markon KE, Patrick CJ, Benning SD, Kramer MD (2007). Linking antisocial behavior, substance use, and personality: an integrative quantitative model of the adult externalizing spectrum. J Abnorm Psychol 116: 645-666.

Levant B, Grigoriadis DE, DeSouza EB (1993). [3H]quinpirole binding to putative D2 and D3 dopamine receptors in rat brain and pituitary gland: a quantitative autoradiographic study. J Pharmacol Exp Ther 264: 991-1001.

Marinelli M, White FJ (2000). Enhanced vulnerability to cocaine self-administration is associated with elevated impulse activity of midbrain dopamine neurons. J Neurosci 20: 8876-8885.

Martinez D, Broft A, Foltin RW, Slifstein M, Hwang DR, Huang Y et al (2004). Cocaine dependence and $\mathrm{d} 2$ receptor availability in the functional subdivisions of the striatum: relationship with cocaineseeking behavior. Neuropsychopharmacology 29: 1190-1202.

Masse LC, Tremblay RE (1997). Behavior of boys in kindergarten and the onset of substance use during adolescence. Arch Gen Psychiatry 54: 62-68. 
Nader MA, Morgan D, Gage HD, Nader SH, Calhoun TL, Buchheimer $\mathrm{N}$ et al (2006). PET imaging of dopamine D2 receptors during chronic cocaine self-administration in monkeys. Nat Neurosci 9: 1050-1056.

Olmstead MC (2006). Animal models of drug addiction: where do we go from here? Q J Exp Psychol (Colchester) 59: 625-653.

Perry JL, Carroll ME (2008). The role of impulsive behavior in drug abuse. Psychopharmacology (Berl) 200: 1-26.

Peterson GB, Ackilt JE, Frommer GP, Hearst ES (1972). Conditioned approach and contact behavior toward signals for food or brain-stimulation reinforcement. Science 177: 1009-1011.

Phillips PE, Robinson DL, Stuber GD, Carelli RM, Wightman RM (2003). Real-time measurements of phasic changes in extracellular dopamine concentration in freely-moving rats by fast-scan cyclic voltammetry. In: Wang JQ (ed). Drugs Of Abuse: Neurological Reviews and Protocols. Totowa, NJ: Human Press. pp 443-464.

Piazza PV, Deminiere JM, Le Moal M, Simon H (1989). Factors that predict individual vulnerability to amphetamine self-administration. Science 245: 1511-1513.

Piazza PV, Deroche-Gamonent V, Rouge-Pont F, Le Moal M (2000). Vertical shifts in self-administration dose-response functions predict a drug-vulnerable phenotype predisposed to addiction. J Neurosci 20: 4226-4232.

Piazza PV, Rouge-Pont F, Deminiere JM, Kharoubi M, Le Moal M, Simon H (1991). Dopaminergic activity is reduced in the prefrontal cortex and increased in the nucleus accumbens of rats predisposed to develop amphetamine self-administration. Brain Res 567: 169-174.

Robinson TE, Berridge KC (1993). The neural basis of drug craving: an incentive-sensitization theory of addiction. Brain Res Rev 18: 247-291.

Robinson TE, Flagel SB (2009). Dissociating the predictive and incentive motivational properties of reward-related cues through the study of individual differences. Biol Psychiatry 65: 869-873.

Roitman MF, Stuber GD, Phillips PE, Wightman RM, Carelli RM (2004). Dopamine operates as a subsecond modulator of food seeking. J Neurosci 24: 1265-1271.

Sandberg SG, Clark JJ, Ng-Evans SB, Parker JG, Phillips PE (2008). Long-term, chronic microsensors for multisite, real-time dopamine detection in behaving animals. In: Society for Neuroscience. Program no. 490.418.2008 Washington, DC.

Seeman P, McCormick PN, Kapur S (2007). Increased dopamine D2(High) receptors in amphetamine-sensitized rats, measured by the agonist $[(3) \mathrm{H}](+)$ PHNO. Synapse 61: 263-267.

Seeman P, Tallerico T, Ko F (2003). Dopamine displaces $[3 \mathrm{H}]$ domperidone from high-affinity sites of the dopamine D2 receptor, but not $[3 \mathrm{H}]$ raclopride or $[3 \mathrm{H}]$ spiperone in isotonic medium: implications for human positron emission tomography. Synapse 49: 209-215.

Seeman P, Weinshenker D, Quirion R, Srivastava LK, Bhardwaj SK, Grandy DK et al (2005). Dopamine supersensitivity correlates with D2 high states, implying many paths to psychosis. Proc Natl Acad Sci USA 102: 3513-3518.

Seiden LS, Andresen J, MacPhail RC (1979). Methylphenidate and d-amphetamine: effects and interactions with alphamethyltyrosine and tetrabenazine on DRL performance in rats. Pharmacol Biochem Behav 10: 577-584.
St Onge JR, Floresco SB (2009). Dopaminergic modulation of riskbased decision making. Neuropsychopharmacology 34: 681-697.

Stead JD, Clinton S, Neal C, Schneider J, Jama A, Miller S et al (2006). Selective breeding for divergence in novelty-seeking traits: heritability and enrichment in spontaneous anxietyrelated behaviors. Behav Genet 36: 697-712.

Stewart J, de Wit H, Eikelboom R (1984). Role of unconditioned and conditioned drug effects in the self-administration of opiates and stimulants. Psychol Rev 91: 251-268.

Stoffel EC, Cunningham KA (2008). The relationship between the locomotor response to a novel environment and behavioral disinhibition in rats. Drug Alcohol Depend 92: 69-78.

Tomie A (1996). Locating reward cue at response manipulandum (CAM) induces symptoms of drug abuse. Neurosci Biobehav Rev 20: 505-535.

Tomie A, Aguado AS, Pohorecky LA, Benjamin D (1998). Ethanol induces impulsive-like responding in a delay-of-reward operant choice procedure: impulsivity predicts autoshaping. Psychopharmacology (Berl) 139: 376-382.

Tomie A, Grimes KL, Pohorecky LA (2008). Behavioral characteristics and neurobiological substrates shared by Pavlovian sign-tracking and drug abuse. Brain Res Rev 58: 121-135.

Uslaner JM, Acerbo MJ, Jones SA, Robinson TE (2006). The attribution of incentive salience to a stimulus that signals an intravenous injection of cocaine. Behav Brain Res 169: 320-324.

Uslaner JM, Dell'Orco JM, Pevzner A, Robinson TE (2008). The influence of subthalamic nucleus lesions on sign-tracking to stimuli paired with food and drug rewards: facilitation of incentive salience attribution? Neuropsychopharmacology 33: 2352-2361.

Uslaner JM, Robinson TE (2006). Subthalamic nucleus lesions increase impulsive action and decrease impulsive choice-mediation by enhanced incentive motivation? Eur J Neurosci 24: 2345-2354.

Uslaner JM, Yang P, Robinson TE (2005). Subthalamic nucleus lesions enhance the psychomotor-activating, incentive motivational, and neurobiological effects of cocaine. J Neurosci 25: 8407-8415.

van Gaalen MM, van Koten R, Schoffelmeer AN, Vanderschuren LJ (2006). Critical involvement of dopaminergic neurotransmission in impulsive decision making. Biol Psychiatry 60: 66-73.

Verbeke G, Molenberghs G (2000). Linear Mixed Models for Longitudinal Data. New York: Springer.

Volkow ND, Fowler JS, Wang GJ, Hitzemann R, Logan J, Schlyer DJ et al (1993). Decreased dopamine D2 receptor availability is associated with reduced frontal metabolism in cocaine abusers. Synapse 14: 169-177.

Weeks J (1972). Long-term intravenous infusion. In: Meyer R (ed). Methods in Psychobiology, London: Academic Press. pp 155-168.

Winstanley CA, Theobald DE, Dalley JW, Cardinal RN, Robbins TW (2006). Double dissociation between serotonergic and dopaminergic modulation of medial prefrontal and orbitofrontal cortex during a test of impulsive choice. Cereb Cortex 16: $106-114$.

Wise RA (2004). Dopamine, learning and motivation. Nat Rev Neurosci 5: 483-494.

Supplementary Information accompanies the paper on the Neuropsychopharmacology website (http://www.nature.com/npp) 